\section{NOTES AND PROBLEMS}

This department welcomes short notes and problems believed to be new. Solutions should accompany proposed problems.

Send all communications concerning this department to

\section{NOTES ET PROBLÈMES}

Cette section a pour but de présenter des notes brèves ainsi que des problèmes inédits. Les problèmes proposés doivent être accompagnés de leurs solutions.

Veuillez adresser les communications concernant cette section à

\author{
I. G. Connell \\ Department of Mathematics \\ McGill University \\ Montreal, Quebec
}

\title{
PROBLEMS FOR SOLUTION
}

This section is dedicated to the memory of Leo Moser who died on February 9, 1970. He was editor of this section during the first three years of the Bulletin (19581960) and always took an active interest in it.

The ten problems listed below were selected by him, when he visited McGill on February 3, from a huge collection he had formed over the years.

P.165. Prove that the set of squares of sides $\frac{1}{2}, \frac{1}{3}, \frac{1}{4}, \ldots$ can be placed without overlap in a square of side $\frac{5}{6}$ but in no smaller square.

P.166. Does there exist a real valued integrable function $f(x)$ which satisfies the conditions:

(i) $f(x)=(f(x))^{2}$;

(ii) $f(x)=f(x+1)$;

(iii) $\int_{0}^{1} f(x) f(x+t) d x=\frac{1}{2}$ for all $t$ ?

P.167. Prove that for every positive integer $n$ and every $\varepsilon>0$ there exists an $N_{0}=N_{0}(n, \varepsilon)$ such that for $N \geq N_{0}$ at least $n$ hyperspheres of diameter $1-\varepsilon$ can be placed without overlap in an $N$-dimensional unit cube. 
P.168. Prove that

$$
\max _{x_{1}^{2}+x_{2}^{2}+x_{3}^{2}=1} \min _{\varepsilon_{i}=0,1,-1(\text { not all } 0)}\left|\varepsilon_{1} x_{1}+\varepsilon_{2} x_{2}+\varepsilon_{3} x_{3}\right|=\frac{1}{\sqrt{21}}
$$

and generalize to the case of more variables.

P.169. What is the largest volume that can be obtained as the cross-section of a four-dimensional unit cube by a hyperplane through its center?

P.170. In the $n$-dimensional tic-tac-toe board let $f(n)$ be the maximal number of "squares" which can be entered without getting three in a line. Prove that $f(n)$ $\geq c 3^{n} / \sqrt{n}$. (Whether $f(n)=o\left(3^{n}\right)$ is unknown.)

P.171. Evaluate

$$
\sum_{(a, b)=1} \frac{1}{a^{2 m} b^{2 n}}
$$

for $m$ and $n$ positive integers. For which values of $m$ and $n$ is the sum rational?

P.172. Let $p(n)$ be the partition function. Prove that for every finite sequence of digits there is an $n$ such that $p(n)$ contains the given sequence of digits in its decimal representation.

P.173. Let $S$ be a measurable point set in the unit square. Suppose no two points of $S$ are at a distance 1/100 (exactly) apart. Prove or disprove that the measure of $S$ is $<\frac{1}{5}$.

P.174. Prove that any measurable subset $S$ of $[0,1]$ having the property that $x, y \in S \Rightarrow(x+y) / 2 \notin S$ has measure 0 .

\section{SOLUTIONS}

P.156. Let $G$ be a group with right invariant metric $d_{R}$. Suppose right multiplication is continuous, then

(i) inversion is continuous at the identity $e$.

(ii) if left multiplication is also continuous, then inversion is continuous everywhere (i.e. $G$ is a topological group);

(iii) if $G$ possesses a left invariant metric $d_{L}$ equivalent to $d_{R}$ then left multiplication is continuous and $G$ is a topological group (equivalent means gives the same topology).

\section{J. MARSDEN,}

University of California at Berkeley 
Solution by A. Finbow, Dalhousie University, Halifax, Nova Scotia. Let $\varepsilon>0$ be a given real number, $x, y \in G$.

(i) $d_{R}(x, e)<\varepsilon \Rightarrow d_{R}\left(x x^{-1}, e x^{-1}\right)<\varepsilon \Rightarrow d_{R}\left(x^{-1}, e\right)<\varepsilon$, by right invariance of $d_{R}$, hence inversion is continuous at $e$.

(ii) Let $x_{0} \in G$, then by continuity of left multiplication there is a real number $\delta>0$ such that $d_{R}\left(x_{0}, x\right)<\delta \Rightarrow d_{R}\left(e, x_{0}^{-1} x\right)<\varepsilon$. But then by the right invariance of $d_{R}, d_{R}\left(x^{-1}, x_{0}^{-1}\right)<\varepsilon$. Hence inversion is continuous everywhere.

To show that $G$ is a topological group, let $x_{0}, y_{0} \in G$. Then by continuity of left multiplication, there is a real number $\delta>0$ such that

$$
d_{R}\left(y_{0}, y\right)<\delta \Rightarrow d_{R}\left(x_{0} y_{0}, x_{0} y\right)<\frac{\varepsilon}{2}
$$

Also by right invariance of $d_{R}$,

$$
d_{R}\left(x_{0}, x\right)<\frac{\varepsilon}{2} \Rightarrow d_{R}\left(x_{0} y, x y\right)<\frac{\varepsilon}{2} .
$$

Then

$$
d_{R}\left(x_{0} y_{0}, x y\right) \leq d_{R}\left(x_{0} y_{0}, x_{0} y\right)+d_{R}\left(x_{0} y, x y\right)<\varepsilon .
$$

(iii) $d_{L}\left(x_{0}, x\right)<\varepsilon \Rightarrow d_{L}\left(g x_{0}, g x\right)<\varepsilon$ for any $g \in G$. Thus since $d_{R}$ and $d_{L}$ are equivalent, left multiplication is continuous and by (ii) $G$ is a topological group.

REMARK. "Suppose right multiplication is continuous" is redundant since $d_{R}(x, y)<\varepsilon \Rightarrow d_{R}(x g, y g)<\varepsilon$ by right invariance of $d_{R}$.

P.162. Let $G$ be a finite abelian group, written additively, and $S$ a subset of $G$. $S$ is said to be a sum-free set in $G$ if $(S+S) \cap S=\varnothing$. Let $\lambda(G)$ denote the largest possible order of a sum-free set in $G$.

For which abelian group $G$ does there exist a sum-free set $S$ such that

(i) $|S|=\lambda(G)$

and (ii) $|S+S|=\frac{\lambda(G)[\lambda(G)+1]}{2}$ ?

A. P. STReet,

University of Alberta

Solution by O. P. Lossers, Technological University, Eindhoven, Netherlands.

(a) Let $C_{l}$ be the cyclic group of order $l$ (represented as the integers mod $l$ ). We suppose $l \geq 4$. Then we can find a positive integer $k$ such that $3(2 k-1)<l \leq 3(2 k+1)$ Now there exists a sum-free set $S$ in $C_{l}$ of $2 k$ elements, namely

$$
S=\{-(2 k-1),-(2 k-3), \ldots,-3,-1,1,3, \ldots, 2 k-3,2 k-1\} .
$$

Hence we deduce:

$$
\frac{\lambda\left(C_{l}\right)}{\left|C_{l}\right|} \geq \frac{2 k}{3(2 k+1)} \geq \frac{4}{15} \text { if } k \geq 2 \text {, i.e. } l \geq 10 .
$$


Direct calculations show that for cyclic groups $C_{l}$ of order $l \leq 9$ the same result holds.

Conclusion: $\lambda(G) \geq \frac{4}{15}|G|$ if $G$ is cyclic.

(b) Let $G$ be a finite abelian group which is not cyclic. We know that $G$ is the direct sum $C \oplus H$ of a cyclic group $C(|C| \geq 2)$ and an abelian group $H$. For every sum-free set $S_{c}$ in $C$ the set $S_{c} \oplus H$ is sum-free in $G$, so that

$$
\lambda(G) \geq \lambda(C) \cdot|H|=\frac{\lambda(C)}{|C|} \cdot|G| .
$$

Conclusion:

$$
\lambda(G) \geq \frac{4}{15}|G| \text { for all finite abelian groups } G .
$$

(c) For each sum-free set $S$ in a group $G$ the following inequality holds trivially: $|S|+|S+S| \leq|G|$. In order that there exist in $G$ a sum-free set $S$ with the required properties (i), (ii), a necessary condition is that

$$
\lambda(G)+\frac{\lambda(G)[\lambda(G)+1]}{2} \leq|G| .
$$

Substitution herein of (1) yields the condition $|G| \leq 16$.

(d) We consider groups $G$ of order $\leq 16$. If $|G|$ is even then $G=C_{2} j \oplus H$ for some positive integer $j$ and abelian group $H$. As $S=\left\{1,3, \ldots, 2^{j}-1\right\}$ is sum-free in $C_{2} j$ we have

$$
\frac{\lambda(G)}{|G|} \geq \frac{\lambda\left(C_{2} j\right)}{\left|C_{2} j\right|} \geq \frac{2^{j-1}}{2^{j}}=\frac{1}{2} .
$$

Now it follows from (2) that $G=C_{2}$. Indeed in $C_{2}$ the set $S=\{1\}$ has the required properties. The (cyclic) groups of order 11 and 13 do not have a sum-free subset with the required properties since in both $\{-3,-1,1,3\}$ is a sum-free set of 4 elements while $4+\frac{1}{2} \cdot 4 \cdot 5>13$. The (cyclic) group of 15 elements $C_{15}=C_{3} \oplus C_{5}$ has a sum-free set of 6 elements since $\lambda\left(C_{5}\right)=2$. Now (2) is not fulfilled: $6+\frac{1}{2} \cdot 6 \cdot 7>15$. The only groups left to consider are $C_{3}, C_{5}, C_{7}, C_{9}$, and $C_{3} \oplus C_{3}$. Direct calculations show:

$$
\begin{array}{llll}
\text { In } C_{3}: & \lambda\left(C_{3}\right)=1, & \text { take } S=\{1\} & \text { then } S+S=\{2\} ; \\
\text { in } C_{5}: & \lambda\left(C_{5}\right)=2, & \text { take } S=\{1,4\} & \text { then } S+S=\{0,2,3\} ; \\
\text { in } C_{7}: & \lambda\left(C_{7}\right)=2, & \text { take } S=\{1,3\} & \text { then } S+S=\{2,4,6\} .
\end{array}
$$

So $C_{3}, C_{5}$ and $C_{7}$ satisfy the conditions. 
$\lambda\left(C_{9}\right)=3$. If there exists in $C_{9}$ a sum-free set $S$ with the required properties the set $S+S$ must contain 0 since $3+\frac{1}{2} \cdot 3 \cdot 4=9$. Without loss of generality we may assume $\{1,8\} \subset S$. But then $S=\{1,3,8\}$ or $S=\{1,6,8\}$, and in both cases $|S+S|=5$ : contradiction.

Finally, $\lambda\left(C_{3} \oplus C_{3}\right) \geq 3$ since $\lambda\left(C_{3}\right)=1$ and again for the sought after sum-free set $S$ we must have $0 \in S+S$. Hence there exist two opposite elements $b$ and $-b$ in $S$. But $b+b=-b$ : contradiction.

ConCLusion: The only groups in which there exists a sum-free set $S$ with the required properties are $C_{2}, C_{3}, C_{5}$, and $C_{7}$.

Also solved by N. Felsinger. 
